\title{
ISABELLE FABRE, Les Vergers de l'âme. L'allégorie du jardin spirituel à la fin du Moyen Âge
}

\section{Paola Cifarelli}

\section{(2) OpenEdition}

\section{Journals}

\section{Édition électronique}

URL : https://journals.openedition.org/studifrancesi/43579

DOI : $10.4000 /$ studifrancesi.43579

ISSN : 2427-5856

\section{Éditeur}

Rosenberg \& Sellier

\section{Édition imprimée}

Date de publication : 1 juin 2021

Pagination : 197-199

ISSN : 0039-2944

\section{Référence électronique}

Paola Cifarelli, «ISABELLE FABRE, Les Vergers de l'âme. L'allégorie du jardin spirituel à la fin du Moyen Âge »,

Studi Francesi [En ligne], 193 (LXV | I) | 2021, mis en ligne le 01 juillet 2021, consulté le 15 octobre

2022. URL : http://journals.openedition.org/studifrancesi/43579 ; DOI : https://doi.org/10.4000/ studifrancesi.43579

Ce document a été généré automatiquement le 15 octobre 2022

\section{(c) (i) (9)}

Creative Commons - Attribution - Pas d'Utilisation Commerciale - Pas de Modification 4.0 International - CC BY-NC-ND 4.0

https://creativecommons.org/licenses/by-nc-nd/4.0/ 


\title{
ISABELLE FABRE, Les Vergers de l'âme. L'allégorie du jardin spirituel à la fin du Moyen Âge
}

\author{
Paola Cifarelli
}

\section{RÉFÉRENCE}

ISABELLE FABRE, Les Vergers de l'âme. L'allégorie du jardin spirituel à la fin du Moyen Âge, Paris, Champion, 2019, «Essais sur le Moyen Âge» 69, 624 pp.

1 Cet ouvrage consacré à la métaphore du jardin spirituel dans la littérature française du Moyen Âge tardif et de la première Renaissance est constitué de deux volets; le premier est une étude littéraire et stylistique d'un corpus de textes qui structurent la totalité ou une partie de la narration autour de cette image (Pierre d'Ailly, Jardin amoureux, vers 1400, passé à l'imprimé en 1475 et réimprimé en 1505 par Antoine Vérard, puis remanié par Michel Bougain vers 1510 sous le titre de Jardin spirituel de l'âme dévote; Jean Gerson, Montagne de contemplation, Canticordum et Trilogie mystique, c. 1400-1425; René d'Anjou, Mortifiement de vaine plaisance, 1455; Jean Henry, Jardin de contemplation, c. 1480; Manuel des dames anonyme, 1510; Gabrielle de Bourbon, Fort chasteau, c. 1516; Jean Thenaud, Triumphe des vertuz, c. 1508-1518); le deuxième volet est constitué d'une édition complète ou partielle de cinq œuvres inédites faisant partie du corpus ou étroitement liées à celui-ci, à savoir le Jardin amoureux de Pierre d'Ailly, le court sermon anonyme Sur le jardin de l'âme dévote (1389) et des extraits du Jardin de contemplation de Jean Henry, du Manuel des dames, du Jardin spirituel de l'âme dévote.

2 Les écrits à usage dévotionnel qu'I.F. soumet à une analyse à la fois détaillée et cohérente dans la première partie étaient destinés principalement aux laïcs, et en premier lieu à un public de cour; ils furent produits à une période où les instances de la devotio moderna se conjuguent avec la tradition monastique d'une part, les transformations de l'allégorie courtoise de l'autre. Initiateur du motif, Pierre d'Ailly 
compose son Jardin amoureux de l'âme autour de 1400; l'ouvrage jouit d'un succès considérable, témoigné par les 25 manuscrits et les six éditions imprimées parvenus jusqu'à nous. L'A. montre que l'évêque de Cambrai se démarque de la tradition précédente par l'inscription du motif du jardin spirituel dans une dimension narrative destinée à décrire les transformations de l'âme qui aspire au divin. Les «syntagmes paysagers» traditionnels qu'il utilise deviennent un support pour la méditation, instrument indispensable de l'élévation de l'âme; dans la lignée du Roman de la Rose, mais en opposition nette avec ce texte didactique tant pour les dimensions de l'ouvrage que pour l'objet du désir, ce petit traité fait du jardin l'aboutissement d'un parcours spirituel qui mène à la rencontre avec le Christ. La comparaison avec le Sermon sur le jardin de l'âme dévote conservé sous forme anonyme dans le ms. Bruxelles, KBR 11206-11207, f. 182-186, et avec le traité De natura et dignitate amoris de Pierre d'Ailly permet de saisir la portée théologique de l'œuvre, qui oppose amour divin et amour mondain; le Jardin de l'âme peut donc être défini un «contre-texte» du Roman de la Rose, dans lequel le chant et la musique jouent un rôle primordial, avant tout sur l'écriture et le style. La Chansonnette amoureuse sur laquelle l'ouvrage se termine pour récapituler la conception d'amour-caritas qui le sous-tend est soumise à une analyse stylistique qui en met en évidence l'originalité. Enfin, l'étude des textes qui côtoient le Jardin dans les différents témoins et des résonances qu'ils produisent sur celui-ci aboutit au constat que la «flexibilité matérielle» (p. 80) est la caractéristique principale du Jardin, et plus généralement de l'écriture de la méditation au $\mathrm{xv}^{\mathrm{e}}$ siècle.

3 Souvent confondu par la critique avec l'auteur du Jardin, Jean Gerson composa trois ouvrages qui exploitent la métaphore horticole, mais avec une intention bien différente. I.F. montre qu'à partir de la Montagne de contemplation le Chancelier fragmente le motif afin de le recomposer et de l'intérioriser. Cet ouvrage de dévotion parmi les plus diffusés au Moyen Âge tardif a pour but l'enseignement de la vie contemplative aux non lettrés, qui seront initiés à une contemplation affective; l'étude de l'organisation spatiale montre qu'elle est ascensionnelle et suit trois étapes, à l'intérieur desquelles la métaphore de la montagne est doublée d'une série d'images servant de similitudes, sans jamais se développer en tant qu'allégories: la montagne est définie un «cadre abstrait et un pur lieu de mémoire» (p. 93), tandis que le jardin est le lieu de la méditation. Un parallèle avec d'autres textes homilétiques gersoniens permet de constater que l'image est souvent utilisée sur le plan rhétorique suivant une stratégie discursive inaugurée par le sermon Nimis honorati sunt; l'image du jardin de l'écriture se précise dans un sermon marial (Tota pulchra es) et s'éclaircit à travers le contenu théorique de la pensée de Gerson tel qu'il est exposé dans le De mystica theologia, issu d'une série de leçons universitaires dispensées en 1402-1403: cette comparaison met en évidence l'incidence que la théorie a sur l'écriture au niveau du montage des images, conçues comme outil mémoriel et cognitif nécessaire pour parler du mystère de l'union mystique, mais jamais utilisées comme véhicule d'une allégorie. La Méditation sur l'Ascension prolonge cette perspective en montrant que les images, et particulièrement celles de l'âme-oiseau et de l'envol, sont utilisées pour conférer au texte son rythme de prose d'art et pour véhiculer le contenu doctrinal. Le Canticordum au pelerin achève et rend plus accessible au public laïque l'exposition de la théorie gersonienne de la contemplation, qui résulte d'un processus d'élévation et de conversion visant à résoudre en un chant harmonieux les discordances intérieures produites par les différents affects de l'intériorité humaine. 
4 Une autre étape importante dans l'évolution de la métaphore du jardin spirituel est représentée par le Mortifiement de vaine plaisance de René d'Anjou, récemment édité par G. Roussineau (Genève, Droz, 2015). En ramenant le motif du jardin au cadre de la fiction courtoise, ce prosimètre conjugue approche mondaine et intention spirituelle en un espace fictionnel centré lui aussi sur le jardin, mais suivant un modèle radicalement différent de celui qui structure la production gersonienne. I.F. analyse d'abord trois éléments du paysage: maisonnette de l'âme, pont branlant sur la rivière symbolisant la colère divine, échelle dressée au pied des remparts protégeant une cité infestée de vices; par-delà leur message spirituel, ces trois lieux permettent de déclencher un débat esthétique à propos de l'utilisation de la métaphore ornementale au niveau de l'inventio. L'attention aux détails dans la scène du jardin montre que René se sert de la fiction pour que la vision contemplative résultant de l'exercice de la méditation affective puisse être rendue sensible au lecteur, tandis que les ressources formelles ont surtout la fonction de rendre accessible le contenu théologique. L'analyse de la prière finale, menée en comparaison avec les Soliloques du Pseudo-Augustin, se révèle particulièrement fructueuse pour montrer la technique du «tissage des voix» (p. 206) qui caractérise l'écriture de René dans cet ouvrage.

5 Les chapitres suivants sont consacrés respectivement au Jardin de contemplation de Jean Henry, un chanoine juriste qui adresse son ouvrage aux femmes dévotes, et à une série d'éditions parues entre 1475 et 1516 dans lesquelles l'influence durable de Pierre d'Ailly se manifeste clairement: le passage à l'imprimé du Jardin amoureux de l'âme, d'abord chez Colard Mansion à Bruges sous le titre de Jardin de devotion (1475) et ensuite chez Antoine Vérard (Jardin de vertueuse consolation, c. 1505), est le témoignage de la diffusion du motif du jardin de l'âme auprès d'un public élargi, qui ne se limitera pas aux lecteurs francophones; quant au Manuel des dames anonyme (vers 1510), il confirme cette donnée. L'étude de la réécriture du texte de Pierre d'Ailly par Michel Bougain, parue au moins trois fois entre 1516 et 1531, et du Fort chasteau pour la retraicte de toutes bonnes ames de Gabrielle de Bourbon, transmis par le ms. Paris, Bibliothèque Mazarine 978, puis celle des Triumphes de vertu de Jean Thenaud (1517-1518) achèvent la partie plus littéraire de cet essai.

6 Dans la deuxième partie, l'édition du Jardin de l'âme est fournie en premier; la description codicologique des 25 manuscrits et des 3 éditions anciennes comporte l'indication de la datation, la description matérielle comprenant les éléments décoratifs, l'indication des possesseurs lorsque cela est connu, l'analyse du contenu qui permet de se rendre compte des textes qui accompagnent généralement le petit traité de Pierre d'Ailly. La bibliographie est particulièrement utile pour repérer les études consacrées à chaque témoin. Le philologue reste un peu sur sa faim pour ce qui est des rapports de parenté entre les témoins; ceux-ci font l'objet, dans chaque notice, d'observations très succinctes qu'on aimerait voir supportées par une analyse des variantes, qui sont d'ailleurs reproduites dans leur intégralité dans les apparats critiques. Une tentative de reconstruction du stemma qui aille plus loin que la simple répartition en deux ensembles sur la base de la forme sous laquelle se présente la Chansonnette permettrait également de mieux rendre compte des dynamiques de la tradition du texte; le choix du manuscrit de base (R, Paris, BnF, fr. 25548) aussi, en résulterait justifié de manière plus pertinente. Toujours à propos de l'analyse philologique contenue dans les notices des témoins, on ne saisit pas toujours exactement le sens attribué à des expressions utilisées apparemment comme 
synonymes, telles 'jumeau' ou 'modèle commun' (voir par exemple les notices consacrées aux mss $\mathrm{C}$ et $\mathrm{M}$ ). Quant aux apparats, ils sont répartis en deux sections; la première figure en bas de page et s'organise en deux étages, contenant respectivement les leçons rejetées du manuscrit de base et les variantes significatives des témoins manuscrits. La deuxième a pour objet les variantes des imprimés et «les autres variantes des témoins manuscrits, à l'exclusion des leçons manifestement fautives et des variantes graphiques et morphologiques non significatives» (p. 393). Un glossaire suit le deuxième apparat.

7 Les autres textes inclus dans la deuxième partie de cet ouvrage ne font l'objet que d'une transcription, complète pour le Sermon sur le jardin de l'âme dévote et partielle pour le Jardin de contemplation de Jean Henry, le Manuel des dames anonyme, le Jardin spirituel de l'âme dévote de Michel Bougain. L'A. précise qu'il ne s'agit que d'un appui à la lecture de la première partie, l'édition critique étant l'objet de travaux en cours.

8 Le volume se clôt sur une table des illustrations qui figurent à la fin de la première partie, ainsi que sur un Index nominum et un Index rerum destinés à faciliter une lecture sélective de cette étude riche et originale. 